\title{
Analysis the Impact of Game theory (Pay-off Matrix, Nash Equilibrium) in the High Tech Industry Al - Digital Assistant
}

\author{
Mozammel Bin Motalab \\ Institute of Information Technology (IIT) \\ University of Dhaka \\ Dhaka, Bangladesh
}

\author{
Muktadir Bin Motalab \\ Department of International Business \\ Faculty of Business Studies, University of Dhaka \\ Dhaka, Bangladesh
}

\begin{abstract}
This paper focuses to discuss and analysis the formal application of game theory which require the identity of independent actors, their preferences, their knowledge, strategic acts they are allowed to make. Each independent actor is assumed to be coherent. This game theory is not limited to case analysis rather psychology, tag of war, business, economy etc. The focus is to use the game theory in information technology more specifically in Artificial Inelegance, Digital Assistant solution. Analysis the outcomes of game theory to determine the strategy for market penetration. The study however reveals some challenges such as affordance, access to information, cost advantage and subsidy or support or funding that change the total game of the deployment plan in order not to exacerbate the problem of digital divide.
\end{abstract}

\section{General Terms}

Decision making, Game theory, Payoff Matrix, ChatBot, AI, Nash Equilibrium.

\section{Keywords}

Artificial Intelligence; Game Theory; Nash Equilibrium; Digital Assistant; Decision Analysis

\section{INTRODUCTION}

The basic tool of game theory is the payoff matrix which is a matrix gives the possible outcome of zero-sum game when player A has possible moves and player B moves. The analysis of the matrix in order to determine optimal strategies is the aim of game theory [14].

This is needed to make a decision after payoff matrix analysis. Payoff matrix helps to take the decision by Nash Equilibrium [3] [14] by which organizations can make their best choices as given preferable action of the competing organizations. The concept of game theory is to deviate an organization's decision with respect to other organization's choice and the result of the game is Nash equilibrium in which one or more opponent has their own strategy. Again, the best possible response to the action of the other organization initiate the action of each organizations.

IT services can be adapted to a new culture in a variety of ways ranging from simple build changes to total redesign in the services. Some need for change becomes obvious with relatively little analysis. For example, a cursory analysis of a country will uncover the need to require AI based digital assistance if it uses a different customer service system, or to indicate product simplification when the local level of technologies is not high [1] [2]. Most such superficial changes can be spotted by looking at service use patterns, the economy, and other related culture elements [10].

\section{PROBLEM OVERVIEW}

In 2018 both company focuses on Digital Assistants Solution one of the specific area of Artificial Intelligence. This is a hightech solution for recent trends. The core feature of the systems are designed to deliver an intuitive, automated experience for all digital channels by engaging customers in natural conversations using voice or text [4] [5]. To add a virtual agent to websites, app, or text messaging application, this system offers customers a consistent experience in any digital channel [7]. A familiar voice will answer their request whether it's typed into a computer, tapped on a screen or spoken into a device. A model of digital assistant especially Chatbot is provided in Fig 1 bellow.

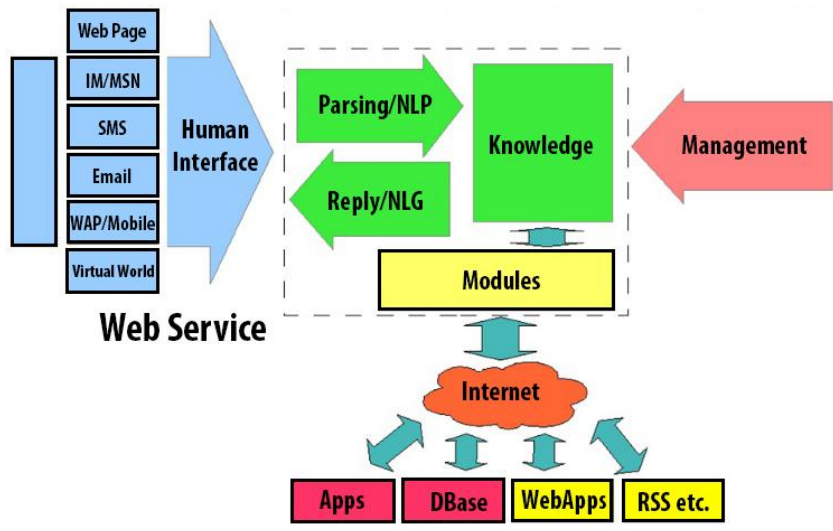

Fig 1 A model of ChatBot

Two company LeadSoft Bangladesh Limited and M Cube Corporation are two Bangladeshi native IT companies working in contemporary system solutions. LeadSoft is a leading Software Development Company, offering custom software development, software products, offshore software development, professional outsourcing and software consultancy. M Cube Corporation has a wide variety of products, ranging from backbone infrastructure to peripheral products, including middleware which are the focus areas of Telecommunication \& Telecom Software Solution providers.

For this Digital Assistant, M Cube Corporation has developed its own platform and want to serve its customer through this. The cost of research and development is huge for the platform development and already exist in the market. Whereas LeadSoft as a partner of Microsoft Corporation, one of the leaders in IT sector an American multinational technology company is using their platform to develop the solution. 


\section{FACTS}

In recent years a new breed of Bangladeshi IT marketers is posing an increasingly credible challenge to go global, following in the footsteps of Japanese and European startup IT to become formidable global competitors. Hi-tech IT solutions among the growing list of international brands making an impact. In this paper, hi-tech defines as Artificial Intelligence, Block Chain, Internet of Things, Business Intelligence and Cloud Computing but not the contemporary software solution like MIS, ERP and IT support services.

Bangladesh governments subsidize high-technology industries (largely focuses on IT) which is expected to create welfares that spillover to other export industries and with a vision to parenting the infant industry. That is, governments believe that high-tech industry produces a positive externality. A strategic advantage to export organizations that are competing with rivals in international markets is subsidies. Subsidies availed by the organizations, bound them to choose between strategic decisions. Strategic interactions between organizations give a sight to capture the strategic decision making by using game theory. Organizations are focusing on actions to exhaust the maximum possibilities of their returns.

\section{PAYOFF MATRIX AND NASH EQUILIBRIUM}

\subsection{Payoff Matrix between Two Firms}

In each quadrant, bottom-left amount shows the revenues of $\mathrm{M}$ Cube, and the upper-right amount shows the revenues of LeadSoft. Here the challenge is each organization necessarily decide whether to deploy a digital assistant or not. A Nash equilibrium is such a condition where each organization is determining its best decision, provided that knowing the actions of other. Considering this condition of payoffs, there exist two state of Nash equilibrium. One of the which is in the upper-right and another one is in bottom-left quadrants. So, it basically means that if LeadSoft deploy then M Cube Corporation does not and if M Cube Corporation does deploy LeadSoft does not.

Table 1. Payoff Matrix - Level Playing Field for Both Firm

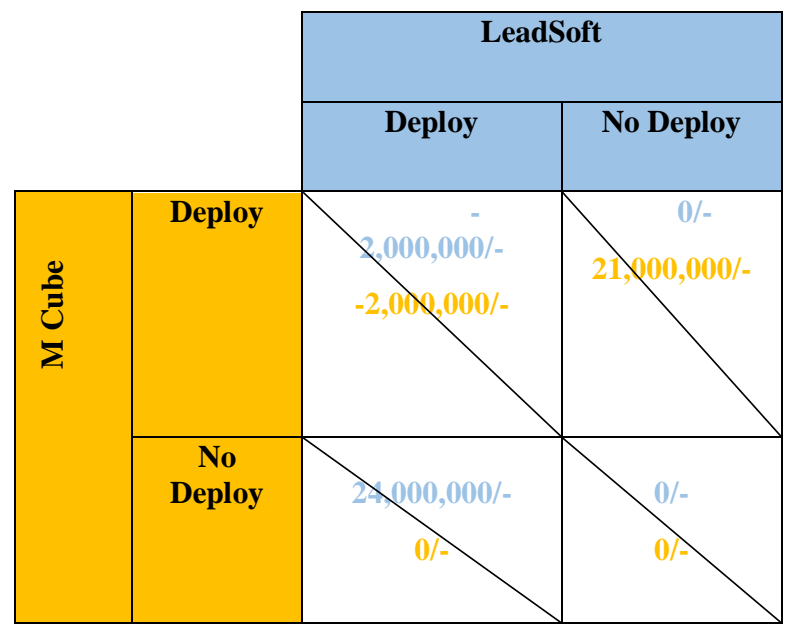

When both the farm are to Deploy then both are going to lose BDT 2,000,000/- . If any of them decided not to deploy the system, then the deploying organization will make a revenue of $21,000,000 /-$ and other will not gain anything. If both the farm decided not to deploy, then both will gain no revenue.

Superlative approach for M Cube If LeadSoft deploys, then M Cube's favorable situation is not to deploy. This outcome demonstrates that having both organizations deploy is not good for any of them and technically it is not a Nash equilibrium. Whenever LeadSoft deploys, $M$ Cube would never be considered in production, since it prefers to leave of the market. Again, finest approach for LeadSoft laying in the decision illustrated in the bottom-left quadrant, where considerably LeadSoft is deploying and $M$ Cube is stay away from deployment. Here this is also a Nash equilibrium, cause each organization is considering its best choice given what is the decision of opponent.

\subsection{Effect of a Subsidy/Support/Fund to LeadSoft}

As LeadSoft is a local company only which can avail any Subsidy or Fund or support by the LICT (Leveraging ICT) Project of Bangladesh Computer Council (BCC) under the Ministry of Post, Telecom and Information Technology of Bangladesh Government. Here is to remember that, M Cube Corporation is registered and headquartered in Singapore.

When the LICT provide a subsidy of BDT 3,000,000/- to LeadSoft, where LeadSoft's revenue increased by that much when it develops the system. There is only one Nash equilibrium identified in the bottom-left quadrant where LeadSoft deploying but $\mathrm{M}$ Cube is not. The revenue for LeadSoft have amplified from BDT 0/- to $24,000,000 /-$ while the subsidy cost is only BDT 3,000,000/-, so there is a net gain of BDT 21,000,000/- in IT industry.

Table 2. Payoff Matrix - Effect of Subsidy/Support/Fund

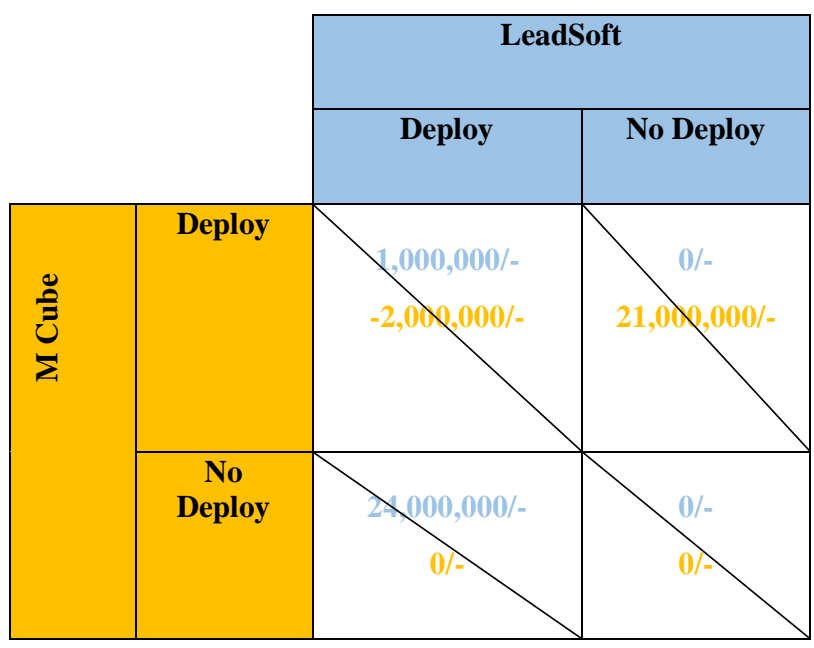

With the subsidy, finest approach for LeadSoft is now to earns BDT $1,000,000 /-$ by producing instead of losing BDT 2,000,000/-. Best strategy for M Cube will want to drop out of the market. Once M Cube makes the decision not to deploy, LeadSoft decision doesn't change. The bottom-left quadrant is a Nash equilibrium where individual organization is determining its best decision along with knowing or exposed actions of the other. Furthermore, it is the only Nash equilibrium.

\subsection{Cost Advantage for $M$ Cube}

M Cube Corporation has their own platform for AI with Digital Assistant which give them a cost advantage of Producing, integrating and deploying the service. Their system is so individual and no dependency on other systems. This platform provides a faster development which $\mathrm{M}$ Cube presume and a technology know how, easily customizable and extendable as well. 
Table 3. Payoff Matrix - - Cost Advantage

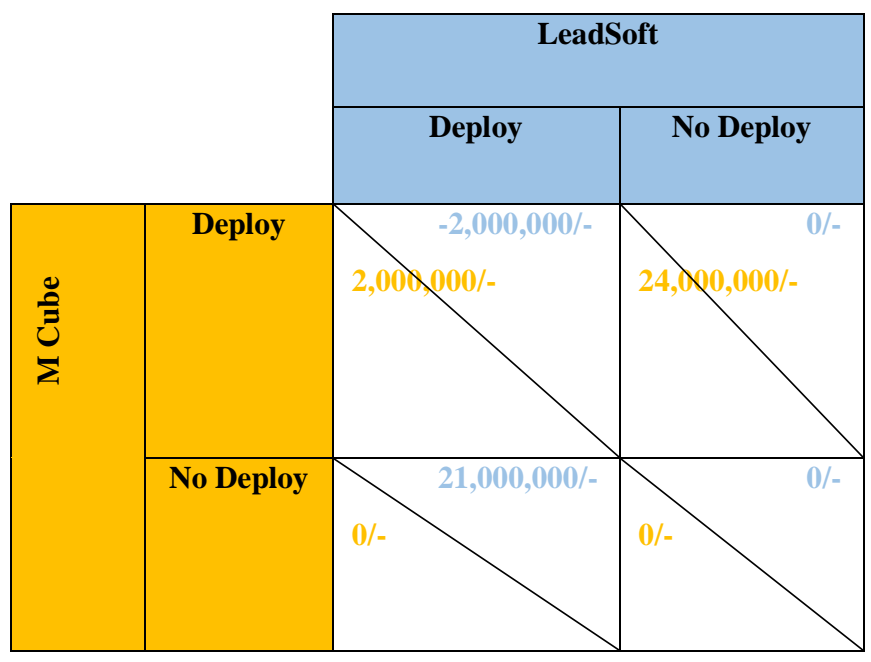

Whereas LeadSoft using Microsoft Bot Framework for their service development. Dependency on Bot Framework may cost in production, integration and dependency.

If $\mathrm{M}$ Cube has a cost advantage in the deployment of Digital Assistant, the payoffs are as shown here. M Cube deploys revenue BDT 2,000,000 when both the organizations are deploying. Again, M Cube making revenue BDT 24,000,000/when LeadSoft does not deploy. Here there is solely one Nash equilibrium which is in the upper-right quadrant. On that specific quadrant M Cube deploys and LeadSoft does not.

\subsection{Subsidy for LeadSoft and Cost Advantages for $M$ Cube}

When the Bangladesh provides a subsidy of BDT 3,000,000/- to LeadSoft, its revenue upsurge by the same volume when it deploys. There is only Nash equilibrium exist which situated in the upper-left quadrant, where both organizations deploy the digital assistance. The revenues for LeadSoft have increased from 0 to BDT $1,000,000 /-$, but the subsidy costs BDT $3,000,000 /-$ million, so there is a net loss of BDT 2,000,000/million in IT industry welfare.

Table 4. Payoff Matrix - Subsidy with Cost Advantage

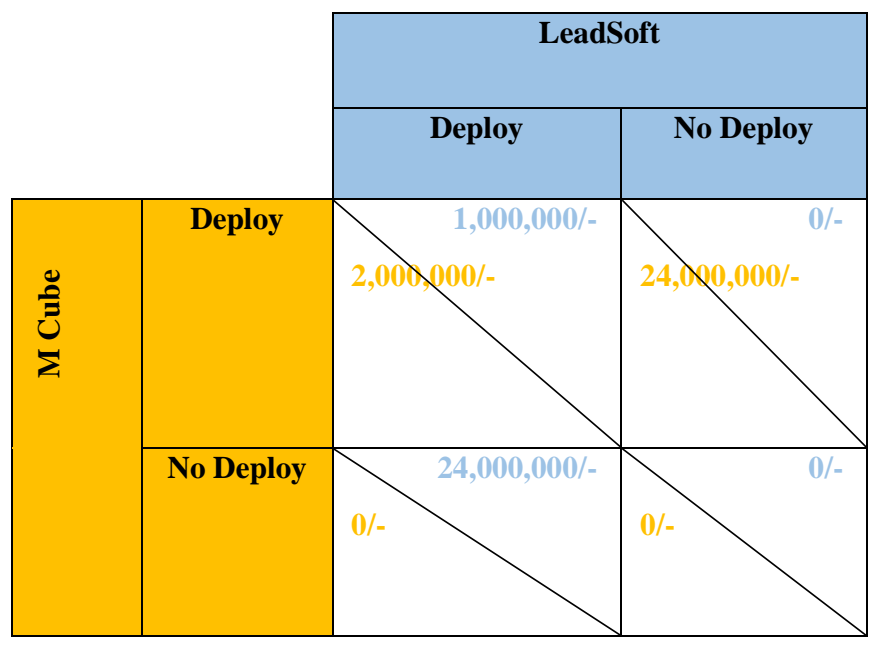

Again, M Cube Corporation has cost advantages of BDT 2,000,000/- as they has their own platform for AI with Digital Assistant. M Cube earns revenues of BDT 2,000,000 when both organizations are producing and revenues of BDT 24,000,000/when LeadSoft does not deploy.

The revenue can be maximized if any of the organization do not lunch their product and other does. Total welfare of the industry in terms of monitory value will be highest in this situation. Nevertheless, Lunching by both organization will not regret or face any potential business loss.

\section{ANALYSIS AND FINDINGS}

- As LeadSoft is availing the LICT fund then of course they are going to deploy the service.

- M Cube Corporation has a leverage of cost effectiveness which convert them to go into the market.

- When there are two Nash equilibrium, there must be some force from outside the model that determines in which equilibrium this is. An example of one such force is the first mover advantage, which means that one organization is able to decide whether or not to deploy before the other organization [14]. Both the company can resolve the issues by present their service firstly in front of customer. This is a healthy competition and both organization can avoid the tag of war.

\section{CONCLUSION}

For a High-tech industry Nash equilibrium is one of the best way to take decision. As M Cube and LeadSoft both are in the same industry, they both need technical support from each other. IT industry touted as crucial First-mover advantage, although now there is a growing backlash against it. First-mover advantage can be instrumental in building market share. In a duopoly Market Strategic decision are required to capture the market. Time to market is also very important. To capture the conflict resolution with strategic decision making of two organizations, by using game theory, the modeling of strategic interactions (games) between organizations as they choose actions that will maximize their returns.

\section{ACKNOWLEDGMENT}

Heartiest gratitude to Dr. Chowdhury Saima Ferdous, Professor, Department of International Business, Faculty of Business Studies, University of Dhaka for her support to us and patronage us to make this write up bring to a different level to make this publishable.

\section{REFERENCES}

[1] Kiner B Shah, Mohit S Shetty, Darshan P Shah and Rajni Pamnani. Approaches towards Building a Banking Assistant. International Journal of Computer Applications 166(11):1-6, May 2017.

[2] Yash Mehta and Shreya Sawkar. The College Chatbot. International Journal of Computer Applications 173(7):34 37, September 2017.

[3] Nash Equilibrium - Columbia University, http://www.columbia.edu/ rs328/NashEquilibrium.pdf

[4] Namita Mhatre, Karan Motani, Maitri Shah and Swati Mali. Article: Donna Interactive Chat-bot acting as a Personal Assistant. International Journal of Computer Applications 140(10):6-11, April 2016. Published by Foundation of Computer Science (FCS), NY, USA.

[5] Gayatri Srivathsan, Asha Bharambe, Hitu Varshney, Poorvi 
Garg and Ramya Bhaskaran. Dialogue Based Assistant for Career Counseling. IJCA Proceedings on International Conference and workshop on Emerging Trends in Technology (ICWET) (13):1-7, 2011.

[6] R. Rajabioun, E. Atashpaz-Gargari and C. Lucas, "Colonial competitive algorithm as a tool for Nash equilibrium point achievement", Lecture Notes in Computer Science, pp. 680695, 2008.

[7] Pauline M. Berry, Melinda Gervasio, Bart Peintner, Neil Yorke-Smith "PTIME: Personalized Assistance for Calendaring"

[8] Hannu Jaakkola and Bernhard Thalheim. (2011), Architecture-driven modelling methodologies, Proceedings of the 2011 conference on Information Modelling and Knowledge Bases XXII . Anneli Heimbürger et al. (eds). IOS Press. p. 98

[9] Bahdanau, D., Cho, K., and Bengio, Y. Neural machine translation by jointly learning to align and translate .arXiv preprint arXiv:1409.0473, 2014.

[10] Abu Shawar B., Atwell E., Different measurement metrics to evaluate a chatbot system, Bridging the Gap: Academic and Industrial Research in Dialog Technologies.

[11] Bot Framework Documentation - Bot Framework, https://docs.microsoft.com/en-us/bot-framework/

[12] Ghosh P, Kalyan B, Sajal D. A game theory-based pricing strategy to support single/multiclass job allocation schemes for bandwidth-constrained distributed computing systems. IEEE T Parall Distr 2007; 18: 289-306.

[13] Harshali Patil and Seema Purohit. Article: Handling Competitiveness in Next Generation Networks using Game Theory. IJCA Proceedings on Emerging Trends in Computing ETC 2016(3):14-17, March 2017.

[14] Robert C. Feenstra; Alan M. Taylor, International Economics - second edition, Chapter 10: Export Subsidies in Agriculture and High-Technology Industries. 